\title{
Aires interfaciales et coefficients de transfert de matière dans un laveur type à jet pulvérisé de liquide dans un Venturi
}

\author{
Interface areas and mass transfer coefficients \\ in a scrubber operating with an atomized jet \\ in a Venturi tube
}

\author{
André Laurent*, Christian Fonteix", \\ Gérard Besson** et Jean-Claude Charpentier* \\ ${ }^{*}$ Laboratoire des Sciences du Génie Chimique (Nancy) \\ ${ }^{*}$ SAPS Anticorrosion
}

\section{Introduction}

Le laveur à jet de liquide dans un venturi est un appareil de plus en plus utilisé comme réacteur gaz-liquide en raison d'un certain nombre de caractéristiques propres qui le rendent souvent indispensable dans un grand nombre de procédés utilisés dans la pratique industrielle et où doivent être mis en contact des produits sous forme liquide et gazeuse.

En effet, le réacteur à jet de liquide dans un venturi est un contacteur gaz-liquide

sans perte de charge et assurant même le plus souvent un effet d'aspiration de gaz par transformation d'une partie de l'énergie du jet,

- autorisant, sans apparition du phénomène d'engorgement, les rapports quantité de liquide sur quantité de gaz très élevés,

- pouvant fonctionner sans trouble dans le cas où le gaz est chargé en poussières solides et jouant même le rôle de dépoussiéreur pour des particules de dimensions supérieures à 4 microns.

Ces propriétés essentielles rarement rencontrées simultanément dans un autre type de réacteur et le nombre limité d'études ou de recherches concernant cet appareil [1 - 4] justifient très largement la mise en œuvre d'une étude permettant la détermination des principaux paramètres de ce matériel.

(*) Laboratoire des Sciences du Génie Chimique C.N.R.S. E.N.S.I.C. 1, rue Grandville, 54042 Nancy.

(**) SAPS Anticorrosion, Le Coudray en Thelle, 60790 Valdampierre.

\section{Principe et description de l'appareil}

Le laveur à jet de liquide pulvérisé dans un venturi est constitué d'un tube contacteur en forme de venturi.

Le liquide est introduit sous forme de jet pulvérisé dans l'axe de l'appareil par une buse fonctionnant sous moyenne pression ( 1 à 5 bars) et assurant l'éjection du liquide à grande vitesse sous forme d'un cône plein de gouttelettes. Toute l'énergie qui va ètre utilisée par le système provient du liquide (débit et pression). Ce dernier est soit renouvelé, soit recyclé par une pompe.

Le gaz est aspiré perpendiculairement à l'axe de l'appareil en partie haute au niveau de l'introduction du liquide. L'énergie du liquide est utilisée pour assurer l'aspiration du gaz et pour disperser le jet de liquide en fines gouttelettes et permettre un contact important entre le gaz et le liquide. Ce contact est par ailleurs amélioré par l'effet de brassage dû aux turbulences au niveau du col du venturi. Cet effet de brassage agit non seulement sur le contact gaz-liquide mais sur le mélange de chaque phase elle-même.

En partie basse du tube venturi, constituant le corps du réacteur et le volume réactionnel proprement dit le mélange gaz-liquide est recueilli dans un bac de pied où se placent les dispositifs à choc permettant la séparation du gaz et du liquide et évitant un entraînement de liquide par le gaz sortant et une capacité de stockage permettant la reprise par une pompe dans le cas le plus fréquent où l'on fonctionne en liquide recyclé.

Le schéma de l'appareillage (fig. 1) représente ce matériel et précise en outre l'ensemble du dispositif de mesure mis en place pour la détermination des débits de gaz et de liquide, des pressions et des tem- 


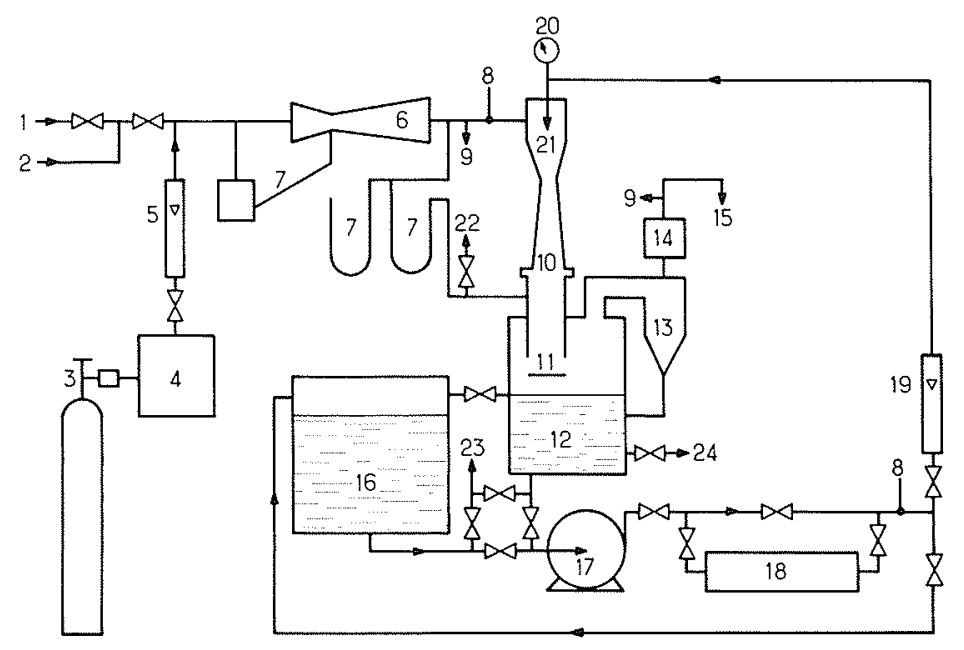

Figure 1. - Schéma de l'appareillage

10 éjecteur venturi
12 bac séparateur
17 pompe
18 échangeur

pératures de gaz et de liquide. Il indique aussi les points de prélèvement et le matériel de maintien en température.

Les dimensions du matériel utilisé (diamètre nominal : $50 \mathrm{~mm}$ ), bien que correspondant à la plus petite réalisation dans la gamme construite, représentent néanmoins un appareil d'utilisation très courante dans la pratique industrielle, en tant que laveur d'évent sur stockage par exemple ou de laveur directement associé à un réacteur dans la chimie fine.

En raison du caractère corrosif des produits qui vont traverser l'appareil en cours d'expérience, l'ensemble est construit en matériaux plastiques à haute tenue chimique : polypropylène pour la pompe et le séparateur et polyester armé pour le venturi, matériaux correspondant ici aussi à la pratique industielle courante.

\section{Hydrodynamique}

\section{Courbes de capacité}

Une partie de l'énergie fournie au système étant transformée en effet d'aspiration du gaz et cette propriété étant l'une des caractéristiques importantes de l'appareil, il a paru utile de déterminer tout d'abord pour différentes valeurs du débit de liquide et de l'énergie mise en jeu les débits de gaz obtenus et à un débit de gaz donné les valeurs de dépression obtenue à l'entrée du venturi.

La figure 2 représente les caractéristiques de pompage de l'ensemble de l'appareil pour différentes valeurs d'énergie mise en jeu sur le liquide. Certains points de ces courbes sont en dehors du domaine d'autoaspiration de l'appareil grâce à un apport de gaz soufflé pour étendre légèrement le domaine étudié.

Il faut noter que pour des rapports du débit volumique de liquide au débit volumique de gaz variant de 0,01 à 0,1 la dépression obtenue à l'entrée de l'appareil varie de 0 à $150 \mathrm{~mm}$ de colonne d'eau.

\section{Taux de rétention}

Le taux de rétention est une caractéristique importante du réacteur. Il a été mesuré en coupant simultanément à un instant donné l'alimentation et l'évacuation en liquide, puis en collectant la quantité de liquide résiduel. Cette mesure est faite dans le venturi seul considéré comme le volume réactionnel. Malgré l'apport élevé de liquide par rapport au gaz, le taux de rétention reste très faible et varie entre 3 et $5 \%$.

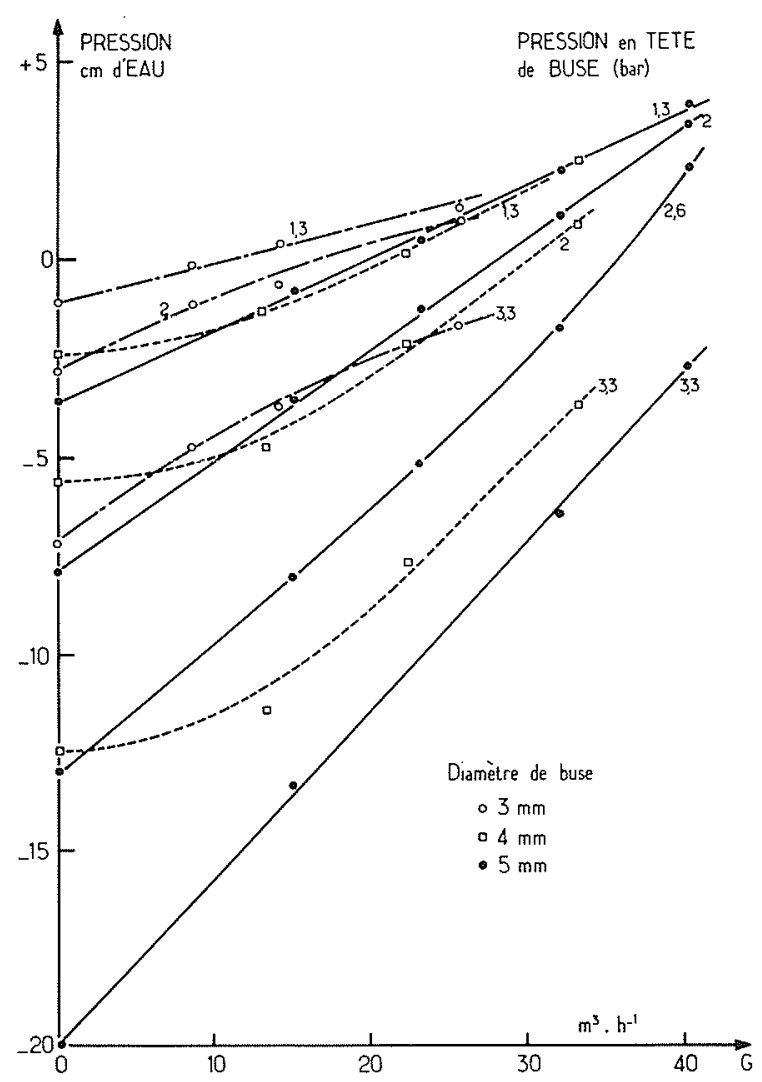

Figure 2. - Courbes de capacité du laveur jet venturi. 


\section{Transfert de matière}

L'aspect transfert de matière du laveur jet venturi est décrit par les paramètres classiques que sont respectivement le produit $k_{G} S$ de la conductance de transfert en phase gazeuse par l'aire interfaciale, le produit $k_{L} S$ de la conductance de transfert en phase liquide par l'aire interfaciale et l'aire interfaciale de contact $S$. Les méthodes utilisées pour la détermination de ces paramètres mettent en cuvre différentes absorptions avec réaction chimique $[5-6]$.

\section{Détermination du produit $k_{G} S$}

Le produit $k_{G} S$ est déterminé par l'absorption avec réaction chimique irréversible et instantanée du dioxyde de soufre dilué dans de l'air par une solution aqueuse d'hydroxyde de sodium.

Dans les conditions de réaction où le contrôle du transfert est uniquement déterminé par la résistance en phase gazeuse, le produit $k_{G} S$ est exprimé par la relation :

$$
k_{G} S=\left(G_{m} / P\right) \cdot \operatorname{Ln}\left(Y_{E} / Y_{S}\right)
$$

La figure 3 représente les résultats obtenus en donnant le produit $k_{G} S$ en fonction du débit de gaz pour différents débits de liquide. Ces courbes montrent que le produit $k_{G} S$ varie dans le même sens que le débit de liquide. La variation avec le débit de gaz est d'autant plus marquée (pente plus importante) que le débit de liquide est plus grand, sauf dans le cas du comportement particulier du laveur équipé d'une buse de $3 \mathrm{~mm}$ de diamètre.

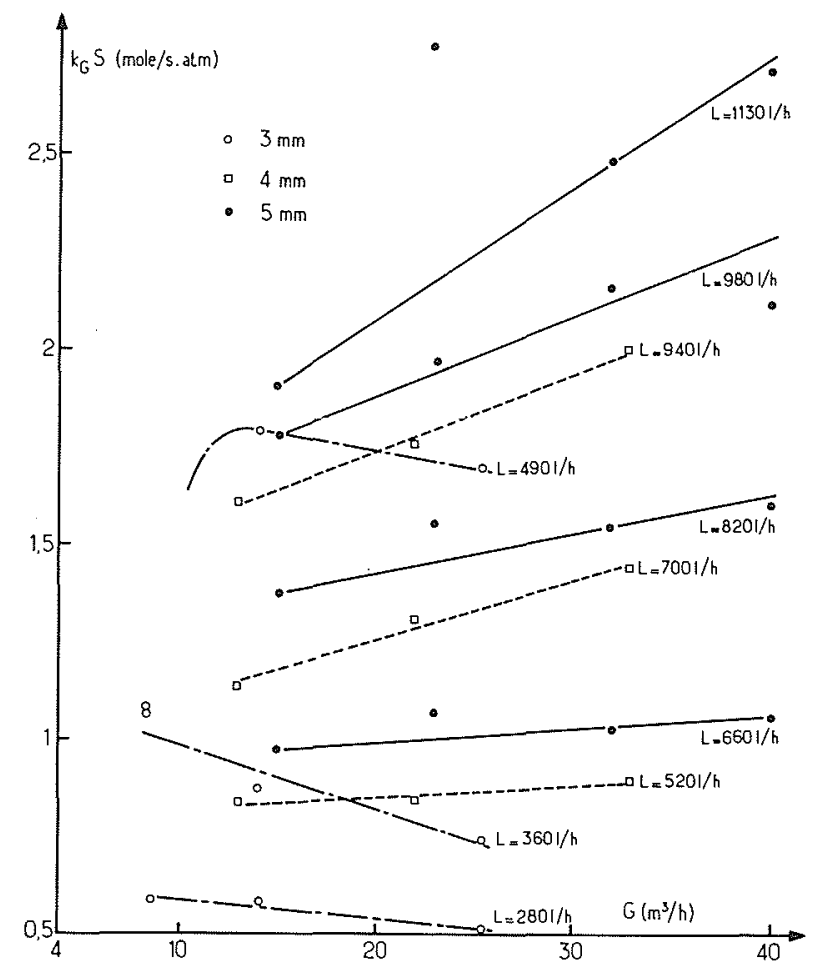

Figure 3. - Variation du produit de la conductance de transfert en phase gazeuse par l'aire interfaciale en fonction des débits de gaz et de liquide.
Ces résultats rendent également compte de l'efficacité de l'appareil dans le cas d'une absorption avec réaction chimique irréversible et instantanée. Dans le domaine de débit étudié, l'efficacité varie de 82 à $99,99 \%$ [7].

\section{Détermination du produit $k_{L} S$}

Le produit $k_{L} S$ est déterminé par l'absorption avec réaction chimique lente du dioxyde de carbone dilué dans l'air par une solution aqueuse de carbonate et de bicarbonate de potassium en présence d'hypochlorite de sodium.

Dans ces conditions, l'expression du produit $k_{L} S$ s'écrit :

$$
k_{L} S=V_{L} \cdot(H e / \dot{y} P) \cdot(d C / d t)
$$

La figure 4 représente les valeurs de $k_{L} S$ obtenues en fonction des débits de gaz et de liquide. Ces courbes montrent que le produit $k_{L} S$ est indépendant du débit de gaz et ne dépend que du débit de liquide dans le domaine d'auto-aspiration de l'appareil. Lorsque l'on sort de ce domaine d'auto-aspiration en envoyant un débit d'air forcé à travers le laveur, le produit $k_{L} S$ augmente alors à la fois avec le débit de gaz et de liquide. L'effet du débit gazeux est d'autant plus marqué que le diamètre de buse est élevé [7 - 8].

\section{Détermination de l'aire interfaciale $S$}

L'aire interfaciale $S$ est mesurée par l'absorption avec réaction chimique en régime rapide du pseudo premier ordre du dioxyde de carbone dilué dans l'air par une solution de soude.

La combinaison de l'expression particulière du flux réactionnel et des équations du bilan différentiel de

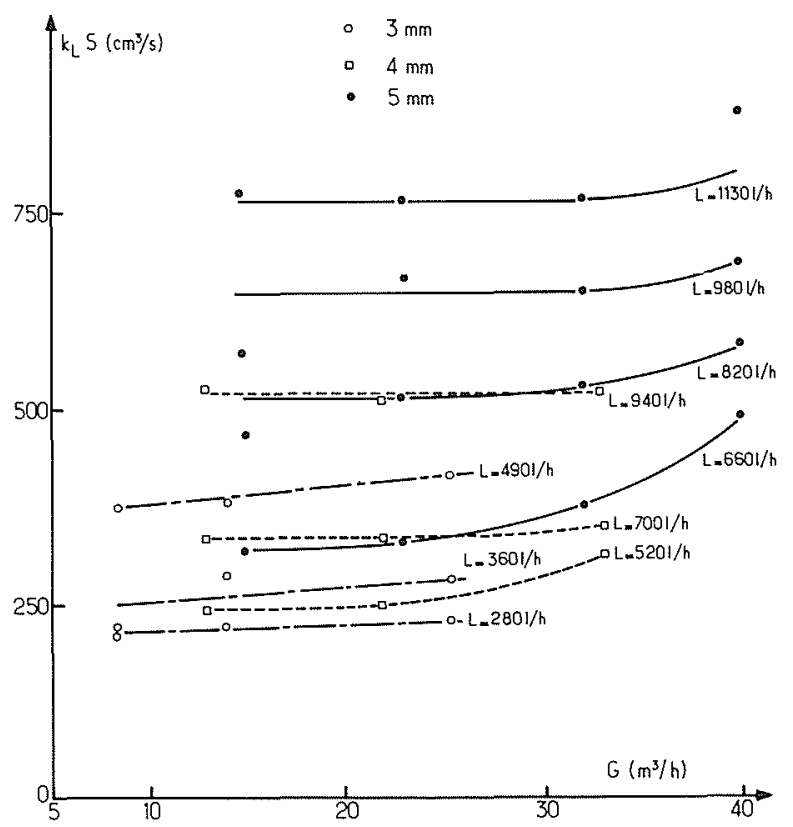

Figure 4. - Variation du produit de la conductance de transfert en phase liquide par l'aire interfaciale en fonction des débits de gaz et de liquide. 


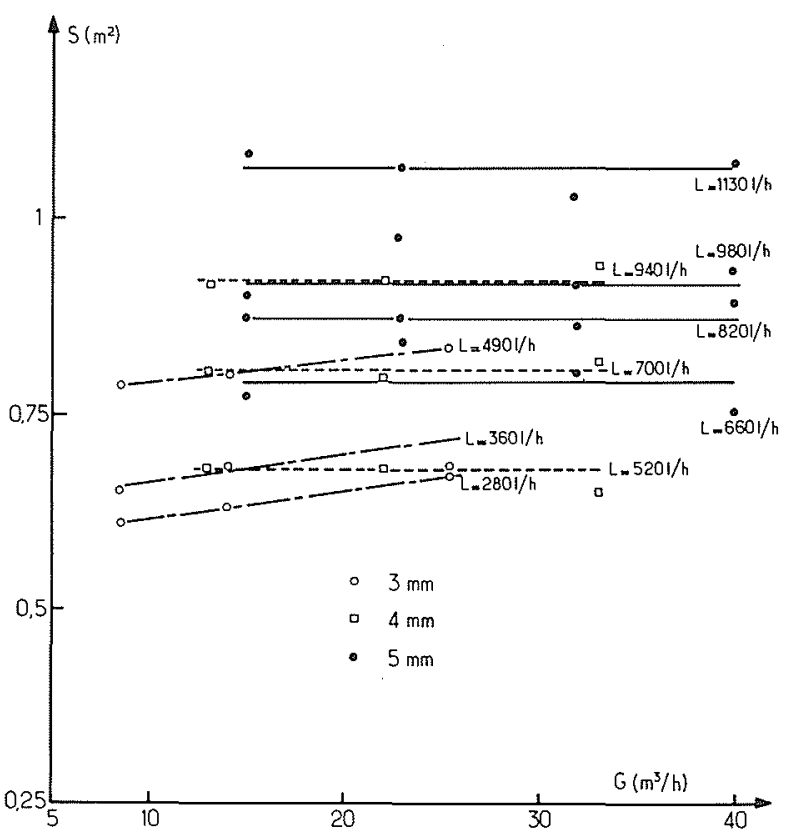

Figure 5. - Variation de l'aire interfaciale en fonction des débits de gaz et de liquide.

matière du laveur conduit à la relation suivante d'estimation de l'aire interfaciale :

$$
S=\left(G_{m} H e / P \sqrt{D_{A} k_{2} C_{B}}\right)\left[\ln \left(Y_{E} / Y_{S}\right)+Y_{\dot{E}}-Y_{S}\right]
$$

Comme la diffusivité et la constante cinétique de la réaction choisie sont connues [ $8-9]$, les mesures respectives des teneurs d'entrée et de sortie en soluté de la phase gazeuse et de la concentration en réactif de la phase liquide permettent donc la détermination de l'aire interfaciale.

La figure 5 représente les valeurs de l'aire interfa. ciale pour différents débits liquides et gazeux. Ces courbes montrent que l'aire interfaciale est pratiquement indépendante du débit de gaz et est une caractéristique de l'appareil pour un débit de liquide donné. Ce résultat est à rapprocher des valeurs de la vitesse de passage du gaz à travers le venturi. Cette vitesse dans le domaine étudié ne dépasse pas $10 \mathrm{~m} / \mathrm{s}$, vitesse insuffisante pour provoquer la rupture des gouttes présentes et par là agir sur la valeur de l'aire interfaciale.
Interprétation et utilisation des résultats obtenus

\section{Comparaison avec d'autres types de contacteur}

Il est intéressant d'interpréter les résultats expérimentaux obtenus en les comparant à des valeurs déjà connues des mêmes paramètres concernant des réacteurs plus classiques.

Les valeurs mesurées et traduites dans les différentes figures ont été ramenées aux unités habituelles, c'est-àdire rapportées à l'unité de volume du réacteur. Le volume du réacteur dont on a tenu compte est celui du tube venturi seul. Des expériences faites simultanément aux expériences ci-dessus par un prélèvement de gaz à la sortie du venturi justifient ce choix en montrant que l'absorption se fait pratiquement en totalité dans le venturi.

Pour le laveur étudié et dans le domaine de débit de liquide et de gaz couvert par l'expérience, on a :

$$
\begin{gathered}
0,14<k_{L} a<0,62 \mathrm{~s}^{-1} ; 4,3<a<7,7 \mathrm{~cm}^{-1} \\
3,5 \cdot 10^{-4}<k_{G} a<19,3 \cdot 10^{-4} \text { mole.cm- } \mathrm{cm}^{-3} \cdot \mathrm{s}^{-1} \cdot \mathrm{atm}^{-1}
\end{gathered}
$$

Le tableau 1 compare ces valeurs à celles déterminées pour quelques autres types de réacteur largement utilisés [10].

La comparaison de ces valeurs va permettre d'utiliser le laveur à jet pulvérisé dans un venturi dans les domaines les plus appropriés.

Notons en passant que les valeurs obtenues avec ce laveur correspondent à une dépense d'énergie de 1,2 à $20,7 \mathrm{~kJ}$ par kilogramme de gaz $[8,11]$.

A titre de comparaison, dans une colonne à garnissage et dans la gamme de valeurs indiquées, la dépense d'énergie va varier de 0,1 à $1,5 \mathrm{~kJ}$ par kilo de gaz traité [17].

Le tableau 1 montre qu'aux faibles valeurs d'énergie mise en jeu dans le laveur jet-venturi, le produit $k_{G} S$ est du même ordre que celui obtenu dans une colonne à garnissage à un niveau d'énergie comparable. L'expérience vient confirmer ces résultats puisque l'on utilise déjà largement ce type de réacteur lorsque l'on se trouve en face d'une absorption avec réaction chimique instantanée.

Ce tableau montre aussi qu'au même niveau d'énergie, les valeurs de $k_{L} S$ restent supérieures à celles ob-

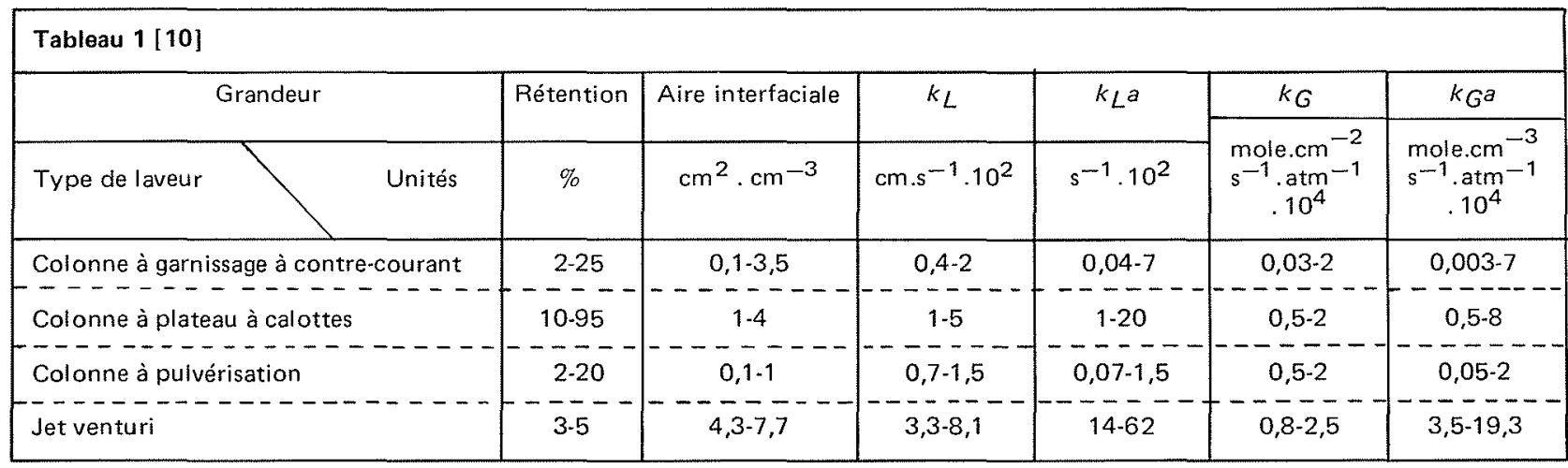




\begin{tabular}{|c|c|c|c|c|c|c|c|c|}
\hline \multicolumn{9}{|l|}{ Tableau 2} \\
\hline \multirow{2}{*}{ Modèle } & \multirow{2}{*}{$\begin{array}{l}\text { Tambour } \\
\text { tournant }\end{array}$} & \multirow{2}{*}{$\begin{array}{l}\text { Ruban } \\
\text { tournant }\end{array}$} & \multirow{2}{*}{$\begin{array}{c}\text { Jet } \\
\text { laminaire }\end{array}$} & \multicolumn{3}{|c|}{ Film tombant } & \multirow{2}{*}{$\begin{array}{l}\text { Colonne à } \\
\text { sphères à } \\
\text { disques }\end{array}$} & \multirow{2}{*}{$\begin{array}{c}\text { Réactêur } \\
\text { agité }\end{array}$} \\
\hline & & & & Sphérique & Cylindrique & Conique & & \\
\hline Temps de contact $(s)$ & $\begin{array}{c}2.10^{-4} \\
10^{-1}\end{array}$ & $\begin{array}{l}6.10^{-4} \\
6.10^{-2}\end{array}$ & $\begin{array}{l}10^{-3} \\
10^{-1}\end{array}$ & $\begin{array}{c}10^{-1} \\
1\end{array}$ & $\begin{array}{c}10^{-1} \\
2\end{array}$ & $\begin{array}{c}2.10^{-1} \\
1\end{array}$ & $\begin{array}{c}10^{-1} \\
2\end{array}$ & $\begin{array}{c}6.10^{-2} \\
10^{-2}\end{array}$ \\
\hline Aire interfaciale $S\left(\mathrm{~cm}^{2}\right)$ & $\begin{array}{c}2 \\
100\end{array}$ & $\begin{array}{l}0,6 \\
6\end{array}$ & $\begin{array}{l}0,3 \\
10\end{array}$ & $\begin{array}{l}10 \\
40\end{array}$ & $\begin{array}{c}10 \\
100\end{array}$ & 80 & $\begin{array}{c}30 \\
360\end{array}$ & $\begin{array}{c}4 \\
80\end{array}$ \\
\hline$k_{L}\left(\mathrm{~cm} \cdot \mathrm{s}^{-1}\right)$ & $\begin{array}{l}0,016 \\
0,356\end{array}$ & $\begin{array}{l}0,021 \\
0,210\end{array}$ & $\begin{array}{l}0,016 \\
0,160\end{array}$ & $\begin{array}{l}0,005 \\
0,016\end{array}$ & $\begin{array}{l}0,0036 \\
0,016\end{array}$ & $\begin{array}{l}0,005 \\
0,011\end{array}$ & $\begin{array}{l}0,0036 \\
0,016\end{array}$ & $\begin{array}{l}0,0016 \\
0,021\end{array}$ \\
\hline $\begin{array}{c}10^{5} k_{G} \\
\left.\text { (mole.cm }{ }^{-2} \cdot \mathrm{atm}^{-1} \cdot \mathrm{s}^{-1}\right)\end{array}$ & - & - & $\begin{array}{l}10 \\
40\end{array}$ & & $1-9$ & & $\begin{array}{r}1 \\
25\end{array}$ & $\begin{array}{r}1 \\
15\end{array}$ \\
\hline$a_{L}=\frac{S}{v_{L}}\left(\mathrm{~cm}^{-1}\right)$ & $\begin{array}{r}100 \\
1250\end{array}$ & $\begin{array}{r}80 \\
400\end{array}$ & $\begin{array}{l}20 \\
80\end{array}$ & $\begin{array}{l}20 \\
60\end{array}$ & $\begin{array}{l}25 \\
60\end{array}$ & $\begin{array}{l}40 \\
70\end{array}$ & $\begin{array}{l}20 \\
60\end{array}$ & $\begin{array}{l}0,002 \\
0,540\end{array}$ \\
\hline
\end{tabular}

tenues dans la colonne à garnissage fonctionnant à contre-courant. Il s'agit d'un phénomène intéressant, mis en lumière par ces expériences puisque jusqu'à ce jour les laveurs à jet ont été rarement utilisés dans le domaine des absorptions avec réaction présentant une résistance au transfert localisée dans la phase liquide.

\section{Simulation du laveur à jet pulvérisé dans un venturi par un réacteur de laboratoire}

La prédiction des performances d'un réacteur gazliquide dans des conditions réactionnelles données n'est que rarement possible. Elle demande en effet soit la connaissance de ces performances dans des conditions similaires à l'échelle pilote ou industrielle, soit celle des caractéristiques de la réaction, c'est-àdire la cinétique, la diffusivité et la solubilité des constituants en présence, ce qui n'est obtenu que pour un nombre encore très limité de systèmes chimiques.

La conduite d'expériences à l'échelle d'un pilote est par ailleurs longue et onéreuse. L'objectif de la tcchnique de simulation consiste à chercher si des appareils simples de laboratoire, faciles à mettre en œuvre et mettant en jeu de faibles quantités de produits ne permettent pas de prédire avec suffisamment de précision le comportement de l'appareil industriel envisagé. Dans l'affirmative, les résultats sont dans ce cas obtenus beaucoup plus rapidement et économiquement.

L'utilisation de modèles expérimentaux de laboratoire a déjà été effectuée avec succès pour la prédiction des performances de colonnes à garnissage industrielles $[12-16]$. On a donc recherché ici d'une part si la méthode pouvait s'appliquer au cas du réacteur à jet liquide pulvérisé dans un venturi et d'autre part à partir de quel appareil de laboratoire.

Des travaux précédents ont montré qu'une simulation est possible si un certain nombre de critères sont respectés entre l'appareil de laboratoire et l'appareil industriel [16]. En résumé, ces critères de simulation impliquent l'égalité des coefficients de transfert $k_{L}$ et $k_{G}$, l'égalité de la grandeur $a_{L}$, aire interfaciale $S$ rapportée au volume de liquide $v_{L}$ dans l'appareil (hold up) et l'égalité des rapports $G / S$ et $L / S$ des débits sur la surface d'échange.

Il y a lieu d'ajouter des critères de simulation des conditions opératoires de fonctionnement, à savoir l'identité des concentrations dans chaque phase et l'identité des conditions de pression et de température.

Selon le type de réaction envisagée, certains critères sont prépondérants et permettent seuls la réalisation de la simulation [8].

Pratiquement, connaissant les valeurs des différents paramètres de l'appareil industriel, le tableau 2 permet de sélectionner le type de réacteur de laboratoire susceptible d'assurer dans des conditions données l'égalité recherchée pour un ou plusieurs critères. Le jet laminaire semblant le mieux convenir a été choisi pour simuler le laveur jet venturi.

Les conditions de fonctionnement à mettre en œuvre dans le modèle de laboratoire pour assurer la simulation résultent de la connaissance des couples de simulation $[8,16]$. Par exemple, les valeurs de $k_{L}$ étant déterminées dans chaque appareil en fonction des débits de liquide, on établit la courbe représentant pour un même $k_{L}$ les débits correspondant dans le jet laminaire et dans le laveur jet venturi, d'où le couple de simulation suivant le critère $k_{L}[8,11,18]$. Le couple de simulation relatif au critère $k_{G}$ est obtenu de la même façon $[8,11]$.

Cette technique de simulation a été appliquée pour différentes conditions opératoires avec quatre systèmes chimiques différents imposant aussi une variation des critères prépondérants de simulation. Le tableau 3 récapitule les conditions mises en œuvre :

\begin{tabular}{|c|c|c|}
\hline \multicolumn{2}{|c|}{ Tableau 3 } & Critères \\
\hline Repère & $\mathrm{CO}_{2}$ - Air - NaOH 0,05 N & $k_{L}$ et $G / S$ \\
A & $\mathrm{CO}_{2}-$ Air - Carbonate $0,6 \mathrm{M}-\mathrm{Bicarbonate}$ & $k_{L}$ et $G / S$ \\
B & $0,2 \mathrm{M}-$ Hypochlorite $0,0176 \mathrm{M}$ & \\
C & $\mathrm{CO}_{2}-$ Air - NaOH 0,5 N & $G / S$ \\
D & $\mathrm{H}_{2} \mathrm{~S}-\mathrm{Air}-$ Diethanolamine $1,2 \mathrm{~N}$ & $k_{G}$ et $G / S$ \\
\hline
\end{tabular}



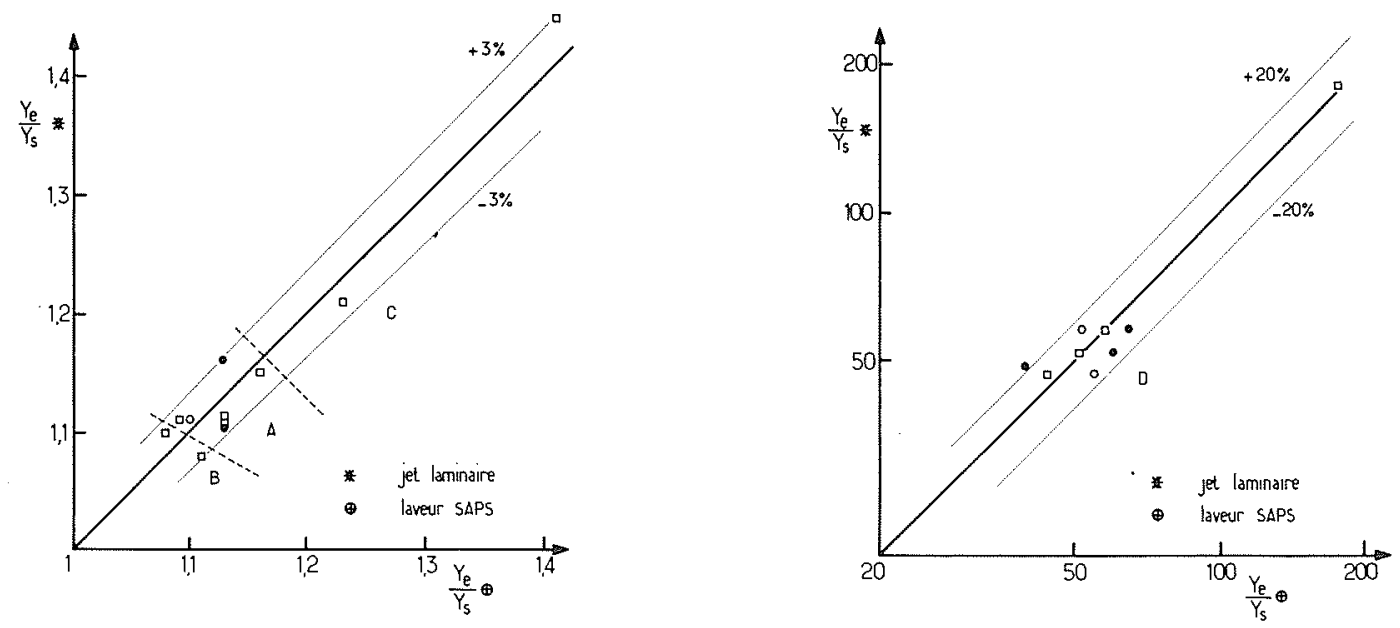

Figure 6. - Comparaison des résultats expérimentaux obtenus dans le laveur-jet ven turi pilote SAPS et par simulation de son fonctionnement par un jet laminaire de laboratoire.

La figure 6 présente l'ensemble des résultats de la simulation du laveur jet venturi par le jet laminaire et permet de se rendre compte de l'estimation de la prévision potentielle de cette technique.

Ces résultats montrent en effet que la méthode proposée semble pouvoir assurer correctement la simulation du réacteur à jet de liquide pulvérisé dans un venturi dans les cas de système réactionnel ayant une cinétique similaire aux systèmes étudiés ci-dessus et dans les limites de débits imposés par les critères de simulation.

\section{Conclusion}

La définition et le dimensionnement d'un réacteur gaz-liquide destiné à assurer une absorption imposée sont encore limités actuellement par les problèmes posés par l'imprécision des corrélations de la littérature, par le manque de données de base permettant leur utilisation et même parfois par l'absence totale de relation utilisable.

L'étude systématique de chaque réacteur accompagnée de la détermination des paramètres permettant la comparaison avec d'autres réacteurs et la mise en place de modèles expérimentaux capables de simuler en laboratoire le matériel industriel, doivent grandement faciliter le choix et la détermination de ce réacteur face à un problème déterminé.

\section{Nomenclature}

a aire interfaciale par unité de volume de réacteur

$a_{L}$ aire interfaciale par unité de volume de liquide

$C$ concentration en produit carbonaté

$C_{B}$ o concentration en réactif

$D_{A}$ diffusivité du soluté dissous dans la phase liquide

$G$ débit volumique de gaz

$G_{m}$ débit molaire de gaz

$\mathrm{He}$ constante de Henry

$k_{G}$ conductance de transfert en phase gazeuse

$k_{L}$ conductance de transfert en phase liquide $k_{2}$ constante de vitesse de la réaction

$L$ débit volumique de liquide

$P$ pression totale moyenne dans le réacteur

$S$ aire interfaciale totale de transfert

$t$ temps

$v_{L}$ hold up volumique dans le venturi

$V_{L}$ volume de liquide total circulant dans l'installation

$\bar{y}$ titre molaire moyen en soluté dans le réacteur

$Y$ rapport molaire en soluté dans la phase gazeuse

\section{Indices}

$E \quad$ entrée

$S$ sortie

\section{Références bibliographiques}

[1] BARKER P.E. - Trans. Instn. Chem. Engrs., 40, 221, 1962.

[2] HARRIS L.S. and HAUN G.R. - Chem. Eng. Progress, 60, $199,1964$.

[3] HOFFMANN R., KURTEN H. und NAGEL O. - Chemie Ing. Techn., 45, 881, 1973.

[4] KROTZSCH P. - Chemie Ing. Techn., 47, 213, 1974.

[5] DANCKWERTS P.V. Gas-liquid reactions, Mc Graw-Hill, New-York, 1970

[6] LAURENT A., PROST C. et CHARPENTIER J.C. - $J$. Chim. Physique, 2, 236, 1975.

[7] LAURENT A., FONTEIX C., BESSON G. and CHARPENTIER J.C. - Atmospheric Pollution 1978, Proceedings of the 13th International Colloquium, Paris, France.

[8] FONTEIX C. - Thèse de Docteur-Ingénieur, Nancy, 1978.

[9] KOLEV N. - Verfahrenstechnik, 7, 3, 71, 1973.

[10] CHARPENTIER J.C. - Advances in chemical engineering, Edited by Vermeulen and Drew, Academic Press, Vol. 11, 1978.

[11] LAURENT A., FONTEIX C., BESSON G. and CHARPENTIER J.C. - Congress CHISA'78, Section Distillation and Absorption, Paper F-2-4, 21-25 August 1978, Praha, Tchecoslovaquie.

[12] DANCKWERTS P.V. and GILLHAM A.J. - Trans. Instn. Chern. Engrs., 44, 42, 1966

[13] LAURENT A., PROST C. and CHARPENTIER J.C. - Chemische Technik, 26, 471, 1974, ref. 52/74.

[14] ALPER E. and DANCKWERTS P.V. - Trans. Instn. Chem. Engrs., 53, 34, 1975. 
[15] ALPER E. and DANCKWERTS P.V. - Chem. Eng. Science, $31,599,1976$

[16] LAURENT A. et Charpentier J.C. - J. Chim. Physique, $10,1001,1977$

[17] HOLZER K. - Chemie Ing. Techn., 51, 200, 1979.
[18] FONTEIX C., LAURENT A, BESSON G. and CHARPENTIER J.C. - Proceedings of the third International Summer School, Modelling of heat and mass transfer processes and chemical reactors, Varna, Bulgarie, May 21-30, 1979.

\section{Discussion}

Le Président. - Je remercie Monsieur BESSON de son exposé. Ce travail résulte d'une collaboration entre la Société SAPS Anticorrosion et le Laboratoire des Sciences du Génie Chimique de Nancy où ont travaillé MM. LAURENT, FONTEIX et CHARPENTIER.

$\mathrm{Y}$ a-t-il des questions au sujet du venturi à jet ?

Comment envisagez-vous l'extrapolation? Vous multipliez les venturis en parallèle ou, au contraire, vous arrivez à faire de très grosses pièces?

M. BESSON. - Les essais ont été faits sur le plus petit appareil industriel. Je n'ai pas donné ses dimensions, mais, pour un débit de gaz maximum de $40 \mathrm{~m}^{3} / \mathrm{h}$, l'entrée de gaz correspondait à une tubulure de $50 \mathrm{~mm}$. C'est le plus petit appareil industriel que nous construisons. Néanmoins, c'est déjà un appareil industriel très utilisé.
Dans la gamme que nous avons pu construire, ces appareils ont une extrapolation parfaite. Nous étions à $40 \mathrm{~m}^{3} / \mathrm{h}$ et nous arrivons jusqu'à $30,40,50000 \mathrm{~m}^{3} / \mathrm{h}$.

Au-desssus, je pense qu'à l'heure actuelle, il vaut mieux passer à des appareils en parallèle, non pas tellement pour le Venturi lui-même, qui peut se construire sans difficulté dans les dimensions bien plus grandes, mais pour les pompes : on va arriver à des débits de plusieurs milliers de $\mathrm{m}^{3} / \mathrm{h}$.

Le Président. - Est-ce que vous réalisez plusieurs étages en série?

M. BESSON. - Cela est fait très couramment mais n'a pas toujours un intérêt évident. Il vaut beaucoup mieux, selon le résultat que l'on a à obtenir, utiliser différents types d'appareils en série.

\section{Abstract \\ Interface areas and mass transfer coefficients in a scrubber operating with an atomized jet in a Venturi tube.}

The working principle and the specific working properties of a venturi-jet scrubber are first described. Then the equipment is presented. The hydrodynamics performances are characterized by an auto aspiration diagram where the aspirated gas flow rate with the corresponding depression at the gas inlet are reported in function of the pressure of the liquid injected at the nozzle. The liquid hold up is also an important parameter.

The mass transfer performances are characterized by the gas-side and liquid-side mass transfer coefficients and the interfacial area. These parameters are determined by the chemical technique of gas absorption with chemical reaction. The influence of the gas and liquid flow rates is shown.

The values of the mass transfer parameters are compared with those of other gas-liquid scrubbers to precise the operation domain of the venturi-jet scrubber and then with those of the laboratory-scale models that could simulate and predict a priori and globally the performance of the present equipment.

The principles of such an integral simulation are briefly recalled and this technique has led to simulate the pilot venturijet scrubber by a laboratory-scale laminar jet within a $20 \%$ accuracy. The results of the simulation experiments are reported. 